\title{
Safety of Major Abdominal Operations in the Elderly: A Study of Geriatric-Specific Determinants of Health
}

\author{
Allison N. Martin $^{1} \cdot$ Darian L. Hoagland ${ }^{1}$ Florence E. Turrentine ${ }^{1,3} \cdot$ R. Scott Jones $^{1,3}$ • \\ Victor M. Zaydfudim ${ }^{1,2,3}$
}

Published online: 21 April 2020

(c) Société Internationale de Chirurgie 2020

\begin{abstract}
Background Preoperative assessment of geriatric-specific determinants of health may enhance perioperative risk stratification among elderly patients. This study examines effects of geriatric-specific variables on postoperative outcomes in patients undergoing elective major abdominal operations.

Methods Patients included in the ACS NSQIP pilot Geriatric Surgery Research File program who underwent elective pancreatic, liver, and colorectal operations between 2014 and 2016 were examined. Multivariable analyses were performed to evaluate associations between patient-specific geriatric variables and risk of death, morbidity, readmission, and discharge destination.

Results A total of 4165 patients were included. Patients $\geq 85$ years were more likely to die, experience postoperative morbidity, and be discharged to a facility (all $p \leq 0.039$ ) than younger patients. Preoperatively, patients $\geq 85$ years were more likely to use a mobility aid, have a prior fall, have consent signed by a surrogate, and to live alone at home prior to operation (all $p<0.001$ ). After adjustment for ACS NSQIP-estimated probabilities of morbidity or mortality, no geriatric-specific preoperative risk factors were significantly associated with increased risk of death or complications in any age group (all $p>0.055$ ). Patients $75-84$ and $\geq 85$ years were more likely to be discharged to facility (OR 2.33 and 4.75 , respectively, both $p<0.001$ ) compared to patients $65-74$ years. All geriatric-specific variables: use of mobility aid, living alone, consent signed by a surrogate, and fall history, were significantly associated with discharge to a facility (all $p \leq 0.001$ ).

Conclusions After adjusting for comorbid conditions, geriatric-specific variables are not associated with postoperative mortality and morbidity among elderly patients; however, geriatric-specific variables are significantly associated with discharge to a facility.
\end{abstract}

Victor M. Zaydfudim

vz8h@virginia.edu

1 Department of Surgery, University of Virginia, Charlottesville, VA, USA

2 Division of Surgical Oncology, Section of Hepatobiliary and Pancreatic Surgery, University of Virginia, Box 800709, Charlottesville, VA 22908-0709, USA

3 Surgical Outcomes Research Center, University of Virginia, Charlottesville, VA, USA

$\begin{array}{ll}\text { Abbreviations } \\ \text { ACS } & \text { American College of Surgeons } \\ \text { ASA } & \text { American Society of Anesthesiology } \\ \text { CI } & \text { Confidence interval } \\ \text { CPT } & \text { Current procedural terminology } \\ \text { GSRF } & \text { Geriatric Surgery Research File } \\ \text { NSQIP } & \text { National Surgical Quality Improvement Program } \\ \text { OR } & \text { Odds ratio } \\ \text { PUF } & \text { Participant use data file } \\ \text { VES-PA } & \text { Vulnerable Elders Surgical Pathways and } \\ & \text { Outcomes Assessment }\end{array}$

Abbreviations

ACS American College of Surgeons

$\mathrm{C}$

GSRF Geriatric Surgery Research File

NSQIP National Surgical Quality Improvement Program

OR Odds ratio

PUF Participant use data file

Outcomes Assessment 


\section{Introduction}

The population of the USA continues to age. Individuals 65 and older are the fastest growing demographic group in the USA, and the portion of this population undergoing general surgery is projected to grow by at least $18 \%$ between 2010 and 2050 [1]. This aging population needs geriatric-specific variables to improve preoperative risk assessment. Preoperative selection and counseling are particularly important in the elective setting [2-4].

A number of recent studies have identified factors associated with perioperative morbidity and mortality in elderly patients [5-7]. Screening tools, such as the Vulnerable Elders Surgical Pathways and Outcomes Assessment (VES-PA) and the comprehensive Optimal Perioperative Management of the Geriatric Patient recommended by the American College of Surgeons (ACS), have been proposed with variable clinical implementation [1]. The complexity of these tools which include multiple components to each assessment system limits their clinical applicability and ability to translate into daily practice $[2,5]$. Age has been independently associated with operative morbidity and mortality in elderly patients $[3,6]$. Frailty has also been associated with perioperative fitness, outcomes, and likely discharge destination [4, 5, 8]. However, many accepted measurements of frailty are also complex [9]. Other proposed variables associated with outcomes in the elderly include do-not-resuscitate status, sarcopenia, and preoperative hemoglobin $[4,5]$.

In 2012, the ACS National Surgical Quality Improvement Program (NSQIP) and the American Geriatrics Society published practice guidelines regarding geriatric preoperative evaluation, proposing that the surgeon assess the patient's individual fitness within nine categories, including nutritional status, frailty, functional status, and mobility among others [10]. Though frailty as a global concept has emerged as a useful predictor of perioperative complications, the most appropriate variables for geriatricspecific risk stratification remain unclear $[2,4,8]$. In 2014, the ACS NSQIP Geriatric Surgery Pilot program began collecting geriatric-specific risk factors, such as cognition, decision making, function, and mobility. Collecting and analyzing data within these new categories allows for targeted investigation of unique risks and outcomes among elderly patients [11]. In this study, we aim to examine the association between patient-specific geriatric variables on short-term postoperative outcomes including mortality, morbidity, readmission, and the impact on discharge destination among patients selected for elective major abdominal operations.
Methods

\section{Patient characteristics and variable definitions}

All patients $\geq 65$ years of age included in the ACS NSQIP Geriatric Surgery Research File (GSRF) between 2014 and 2016 were linked with the ACS Participant Use Data File (PUF) using patients' case ID variable. Both ACS NSQIP PUF and GSRF data files report aggregated, patient-level HIPAA compliant data provided by participating institutions. Analyses of these de-identified public datasets have been designated exempt by the University of Virginia Institutional Review Board for Health Sciences Research. The GSRF dataset during the study period includes data from the pilot phase of the ACS NSQIP Geriatric Surgery Project [12]. This pilot phase included 25 of the 680 (4\%) 2016 ACS NSQIP participating sites. Patients who had operations defined by the ACS NSQIP Procedure Targeted Hepatectomy, Pancreatectomy, Colectomy, and Proctectomy Current Procedural Terminology (CPT) codes (see Appendix) were included in this study. Patients who had emergency operations and those with American Society of Anesthesiology (ASA) classification 5 were excluded.

Clinical covariates extracted from the PUF dataset included age, sex, race/ethnicity, ASA classification, functional status (categorized/recoded as independent or dependent), operation (as defined by CPT codes), and ACS NSQIP-estimated probability of mortality and morbidity. ACS NSQIP-estimated probabilities of mortality and morbidity are calculated for each individual patient using hierarchical regression models that account for multiple patient-level factors included in the PUF dataset [13]. These probabilities represent the estimated likelihood that an individual patient will experience mortality or composite morbidity outcome during the ACS NSQIP-defined follow-up period and are particularly useful in statistical adjustment of clinically relevant comorbid conditions. Clinical geriatric-specific variables extracted from the GSRF dataset included patient origin status (categorized as living home alone, home with companion, or residing at a facility), use of mobility aid, patient fall history (categorized as fall within the past year or no falls), and patient competency to sign own consent.

\section{Outcome measures}

Primary outcome measures were ACS NSQIP-defined mortality and composite morbidity. Both were defined as event occurrence within 30 days following the operation or during the index hospitalization. Composite ACS NSQIP morbidity definition includes pneumonia; reintubation; failure to wean off the ventilator within $48 \mathrm{~h}$; renal 
insufficiency; renal failure; cardiac arrest; myocardial infarction; stroke; sepsis; septic shock; fascial dehiscence; superficial, deep, or organ-space surgical site infection; urinary tract infection; bleeding; perioperative blood transfusion; and venous thromboembolism (including deep venous thrombosis and/or pulmonary embolus).

Secondary outcome measures included unplanned hospital readmission and patient discharge destination. Readmission was defined as unplanned hospital readmission within 30 days of the operation. Discharge destination was stratified as home versus discharge to any other facility.

\section{Statistical analyses}

Categorical variables were reported as frequencies (percent); age is reported as median (interquartile range) and as a categorical variable age group (65-74, 75-84, and $\geq 85$ ) in consistency with the current ACS NSQIP data definitions for the inclusion in the multivariable models. Differences in distribution of categorical variables were compared using Chi square or Fisher's exact test, as appropriate. Four separate multivariable models were developed to test the associations between a priori determined relevant covariates and primary and secondary outcome measures. Covariates included in multivariable models were age group, hepatopancreatic versus colorectal operation, and logit of the ACS NSQIP-estimated probability of mortality (for mortality model) or logit of the ACS NSQIP-estimated probability of morbidity (for morbidity, readmission, and discharge destination models). Patients who died were excluded from the readmission and discharge destination multivariable models. All four geriatricspecific GSFR variables were included in each of the multivariable models. Importantly, as already indicated, patient's functional status is included in the PUF dataset and is a covariate in the ACS NSQIP-estimated probabilities of morbidity and mortality. All data management and statistical analyses were performed using STATA version 14.2 (StataCorp LP, College Station, Texas).

\section{Results}

A total of 4165 patients were included; median age 73 years (IQR 68-78); 54\% female; 79\% white. A total of 780 patients (19\%) had pancreatic resections; 387 patients (9\%) had liver resections; and 2998 patients (72\%) had colon or rectal resections. A total of 339 patients (8\%) were 85 years of age or older. Patient demographic and clinical covariates, geriatric-specific variables, and outcomes measures stratified by age group are summarized in Table 1. Patients 85 years or older were more likely to have a prior history of fall, utilize mobility aids, have surgical consent signed by a surrogate, and either to live at home alone or live at a facility compared to younger patients (all $p<0.001$ ). Patients 85 years or older were more likely to die, more likely to experience postoperative morbidity, and were more likely to be discharged to a facility compared to younger patients (all $p \leq 0.039$ ). Postoperative complications by age group are summarized in Table 2. There was no difference in patient readmission between the three age groups $(p=0.686)$.

A total of 78 patients $(1.9 \%)$ died in the postoperative period: $30(1 \%)$ patients $65-74$ years, 25 (2\%) patients 75-84 years, and $18(5 \%)$ patients 85 years and older $(p<0.001)$. After adjustment for significant effect of ACS NSQIP-estimated probability of mortality (odds ratio (OR) $2.43,95 \%$ confidence interval (CI): 1.95-3.04, $p<0.001$ ), none of the geriatric-specific preoperative risk factors were associated with increased risk of mortality (all $p \geq 0.071$, Table 3). A total of 708 patients (17\%) had ACS NSQIPdefined composite morbidity during the postoperative period: $394(16 \%)$ patients 65-74 years, 246 (19\%) patients $75-84$ years, and $68(20 \%)$ patients 85 years and older $(p=0.039)$. After adjustment for significant effect of ACS NSQIP-estimated probability of morbidity (OR 3.71, 95\% CI 3.06-4.51, $p<0.001$ ), none of the geriatricspecific preoperative risk factors were associated with increased risk of morbidity (all $p \geq 0.055$, Table 4).

A total of 496 patients (12\%) had ACS NSQIP-defined unplanned readmission during the postoperative period: $306(13 \%)$ patients $65-74$ years, $150(12 \%)$ patients $75-84$ years, and $40(12 \%)$ patients 85 years and older $(p=0.686)$. After adjustment for significant effect of ACS NSQIP-estimated probability of morbidity (OR 2.19, $95 \%$ CI $1.76-2.71, p<0.001$ ), only consent signed by a surrogate was associated with an increased rate of readmission (OR1.75, 95\% CI 1.01-3.04, $p=0.046$ ). The remainder of the geriatric-specific preoperative risk factors were not associated with increased rate of readmission (all $p$ $\geq 0.078$, Table 5). A total of 734 patients (18\%) were discharged to a facility after index operation: $252(10 \%)$ patients 65-74 years, 315 (24\%) patients 75-84 years, and $150(47 \%)$ patients 85 years and older. After adjusting for significant effects of other covariates, all geriatric-specific variables were independently associated with discharge to a facility (all $p \leq 0.001$, Table 6). Three geriatric-specific variables were associated with greater likelihood of discharge to a facility other than home: preoperative use of mobility aid (OR 2.30, 95\% CI 1.85-2.85, $p<0.001$ ), preoperative fall history (OR 1.56, 95\% CI 1.19-2.05, $p=0.001$ ), and consent signed by a surrogate (OR 4.71, 95\% CI 2.78-7.97, $p<0.001)$. Patients who lived at home with a companion were less likely than patients who lived home alone to be discharged to a facility (OR $0.41,95 \% \mathrm{CI}$ $0.33-0.50, p<0.001)$. 
Table 1 Demographic and clinical covariates stratified by age group

\begin{tabular}{|c|c|c|c|c|}
\hline & $\begin{array}{l}65-74 \text { years } \\
n=2466\end{array}$ & $\begin{array}{l}75-84 \text { years } \\
n=1319\end{array}$ & $\begin{array}{l}\geq 85 \text { years } \\
n=339\end{array}$ & $p$ value \\
\hline Female sex & $1293(52)$ & $707(54)$ & $219(65)$ & $<0.001$ \\
\hline Racelethnicity & & & & 0.016 \\
\hline White & $1939(82)$ & $1054(86)$ & $282(89)$ & \\
\hline Black & $262(11)$ & $108(9)$ & $22(7)$ & \\
\hline Hispanic & $101(4)$ & $44(4)$ & $12(4)$ & \\
\hline Other & $36(2)$ & $24(2)$ & $1(<1)$ & \\
\hline ASA class & & & & $<0.001$ \\
\hline Class 1 & $15(<1)$ & $3(<1)$ & $1(<1)$ & \\
\hline Class 2 & $796(32)$ & $308(23)$ & $50(15)$ & \\
\hline Class 3 & $1550(63)$ & $895(68)$ & $245(72)$ & \\
\hline Class 4 & $103(4)$ & $111(8)$ & $43(13)$ & \\
\hline Operation & & & & $<0.001$ \\
\hline Pancreatic & $509(21)$ & $244(19)$ & $26(8)$ & \\
\hline Liver & $253(10)$ & $127(10)$ & $6(2)$ & \\
\hline Colorectal & $1704(69)$ & $948(72)$ & $307(91)$ & \\
\hline Dependent functional status & $50(2)$ & $68(5)$ & $44(13)$ & $<0.001$ \\
\hline Mobility aid & $282(11)$ & $310(24)$ & $160(47)$ & $<0.001$ \\
\hline Fall history & $177(7)$ & $168(13)$ & $67(20)$ & $<0.001$ \\
\hline Patient can sign own consent & $2424(98)$ & $1280(97)$ & $305(90)$ & $<0.001$ \\
\hline Patient origin status & & & & $<0.001$ \\
\hline Home with companion & $1886(78)$ & $923(72)$ & $189(58)$ & \\
\hline Home alone & $523(22)$ & $361(28)$ & $128(40)$ & \\
\hline Facility & $5(<1)$ & $5(<1)$ & $7(2)$ & \\
\hline ACS NSQIP probability of morbidity & $15.2(10.3-22.9)$ & $17.3(11.7-24.8)$ & $17.8(10.7-25.7)$ & $<0.001$ \\
\hline ACS NSQIP probability of mortality & $0.6(0.3-1.4)$ & $1.2(0.6-2.7)$ & $2.2(1.1-4.9)$ & $<0.001$ \\
\hline Death & $30(1)$ & $25(2)$ & $18(5)$ & $<0.001$ \\
\hline Composite complications & $394(16)$ & $246(19)$ & $68(20)$ & 0.039 \\
\hline Readmission & $308(12)$ & $154(12)$ & $43(13)$ & 0.742 \\
\hline Discharge to destination other than home & $276(11)$ & 337 (26) & $164(48)$ & $<0.001$ \\
\hline
\end{tabular}

ACS NSQIP American College of Surgeons National Surgical Quality Improvement Program, ASA American Society of Anesthesiology

\section{Discussion}

This analysis of geriatric-specific variables demonstrates strong and significant association between clinical factors associated with increasing age and frailty and postoperative destination to a facility other than home. Over a quarter of patients older than 75 years and nearly half of patients over 85 years were discharged to a facility other than home after elective hepatopancreatic or colorectal resection. Specifically, all geriatric-specific variables including preoperative use of mobility aid, preoperative falls, consent signed by a surrogate, and living at home without a companion were associated with discharge to a facility in this patient population.
These geriatric-specific variables, however, were not associated with postoperative mortality and morbidity; consent signed by a surrogate was the only geriatricspecific variable associated with readmission. ACS NSQIP probabilities of mortality and morbidity were the only statistically significant variables associated with postoperative death and complications. These probabilities were calculated from the clinically meaningful comorbid factors included in the ACS NSQIP calculator and available for preoperative risk estimation for any individual patient. The specific number and type of variables included fluctuate year to year and differ between probability of morbidity and probability of mortality variables. For example, the 2015 mortality model was derived from 404,564 patient records (1.15\% 30-day NSQIP-defined mortality) and 
Table 2 Postoperative complications stratified by age group

\begin{tabular}{|c|c|c|c|c|}
\hline Complication & $\begin{array}{l}65-74 \text { years } \\
n=2466\end{array}$ & $\begin{array}{l}75-84 \text { years } \\
n=1319\end{array}$ & $\begin{array}{l}\geq 85 \text { years } \\
n=339\end{array}$ & $p$ value \\
\hline Pneumonia & $59(2.4)$ & $53(4.0)$ & $16(4.7)$ & 0.004 \\
\hline Reintubation & $59(2.4)$ & $43(3.3)$ & $9(2.7)$ & 0.283 \\
\hline Failure to wean ventilator & $42(1.7)$ & $20(1.5)$ & $7(2.1)$ & 0.715 \\
\hline MI & $17(0.7)$ & $14(1.1)$ & $6(1.8)$ & 0.100 \\
\hline Cardiac arrest & $19(0.8)$ & $14(1.1)$ & $3(0.9)$ & 0.657 \\
\hline Bleeding & $252(10)$ & $158(12)$ & $56(17)$ & 0.002 \\
\hline Transfusion & $33(1.3)$ & $37(2.8)$ & $15(4.4)$ & $<0.001$ \\
\hline DVT & $34(1.4)$ & $25(1.9)$ & $10(3.0)$ & 0.080 \\
\hline $\mathrm{PE}$ & $16(0.7)$ & $14(1.1)$ & $3(0.9)$ & 0.391 \\
\hline Sepsis & $85(3.5)$ & $57(4.3)$ & $11(3.2)$ & 0.368 \\
\hline Septic shock & $46(1.9)$ & $31(2.4)$ & $12(3.5)$ & 0.120 \\
\hline SSSI & $123(5.0)$ & $57(4.3)$ & $10(3.0)$ & 0.217 \\
\hline DSSI & $19(0.8)$ & $10(0.8)$ & $5(1.5)$ & 0.371 \\
\hline OSSI & $154(6.2)$ & $70(5.3)$ & $15(4.4)$ & 0.289 \\
\hline Fascial dehiscence & $21(0.9)$ & $11(0.8)$ & $1(0.3)$ & 0.551 \\
\hline Renal insufficiency & $22(0.9)$ & $14(1.1)$ & $4(1.2)$ & 0.808 \\
\hline Acute renal failure & $14(0.6)$ & $6(0.5)$ & $1(0.3)$ & 0.759 \\
\hline Urinary tract infection & $64(2.6)$ & $49(3.7)$ & $11(3.2)$ & 0.143 \\
\hline Stroke & $6(0.2)$ & $7(0.5)$ & $3(0.9)$ & 0.123 \\
\hline
\end{tabular}

Summation of individual complications for each age group exceeds composite complications value for individual age groups reported in Table 1, as many patients had more than one complication

$M I$ myocardial infarction, $D V T$ deep venous thrombosis, $P E$ pulmonary embolus, SSSI superficial surgical site infection, DSSI deep surgical site infection, OSSI organ-space surgical site infection

Table 3 Multivariable mortality model incorporating geriatric-specific variables

\begin{tabular}{lll}
\hline & OR & $95 \%$ CI \\
\hline Age & & \\
$65-74$ & Reference & $0.45-1.47$ \\
$75-84$ & 0.82 & $0.76-3.15$ \\
85 and older & 1.55 & 0.501 \\
Operation & & 0.230 \\
Hepatopancreatic & Reference & $0.51-1.55$ \\
Colorectal & 0.89 & $1.95-3.04$ \\
ACS NSQIP-estimated probability of mortality & 2.43 & $0.46-1.55$ \\
Mobility aid & 0.85 & $0.95-3.33$ \\
Fall history & 1.78 & 0.689 \\
Consent & & $<0.001$ \\
Signed by patient & Reference & 0.590 \\
Surrogate & 2.04 & 0.071 \\
Patient origin & & $0.84-4.96$ \\
Lives home alone & Reference & \\
Lives with companion & 0.77 & 0.118 \\
Lives at facility & 0.64 & $0.45-1.35$ \\
\hline ACS NSIP Ame & $0.072-5.67$
\end{tabular}

ACS NSQIP American College of Surgeons National Surgical Quality Improvement Program 
Table 4 Multivariable morbidity model incorporating geriatric-specific variables

\begin{tabular}{lll}
\hline & OR & $95 \%$ CI \\
\hline Age & Reference & \\
$65-74$ & 1.02 & $0.84-1.23$ \\
$75-84$ & 1.08 & $0.78-1.49$ \\
85 and older & & 0.850 \\
Operation & Reference & 0.650 \\
Hepatopancreatic & 1.09 & $0.90-1.33$ \\
Colorectal & 3.71 & $3.06-4.51$ \\
ACS NSQIP-estimated probability of morbidity & 1.24 & $1.00-1.55$ \\
Mobility aid & 1.09 & $0.83-1.43$ \\
Fall history & & 0.385 \\
Consent & Reference & $<0.001$ \\
Signed by patient & 1.33 & 0.055 \\
Surrogate & & 0.550 \\
Patient origin & Reference & $0.81-2.20$ \\
Lives home alone & 1.13 & 0.260 \\
Lives with companion & 2.09 & $0.92-1.38$ \\
Lives at facility & Improvent Progran & $0.72-6.06$ \\
\hline ACS NSP Ameris
\end{tabular}

ACS NSQIP American College of Surgeons National Surgical Quality Improvement Program

Table 5 Multivariable unplanned readmissions model incorporating geriatric-specific variables

\begin{tabular}{lll}
\hline & OR & $95 \%$ CI \\
\hline Age & Reference & \\
$65-74$ & 0.82 & $0.66-1.02$ \\
$75-84$ & 0.89 & $0.61-1.30$ \\
85 and older & & 0.078 \\
Operation & Reference & 0.544 \\
Hepatopancreatic & 0.96 & $0.77-1.20$ \\
Colorectal & 2.19 & $1.76-2.71$ \\
ACS NSQIP-estimated probability of morbidity & 1.16 & $0.90-1.50$ \\
Mobility aid & 1.00 & $0.72-1.38$ \\
Fall history & & 0.726 \\
Consent & Reference & $<0.001$ \\
Signed by patient & 1.75 & 0.253 \\
Surrogate & & 0.997 \\
Patient origin & Reference & $1.01-3.04$ \\
Lives home alone & 1.14 & 0.046 \\
Lives with companion & 1.14 & $0.91-1.44$ \\
Lives at facility & $0.31-4.24$
\end{tabular}

ACS NSQIP American College of Surgeons National Surgical Quality Improvement Program

includes 31 distinct covariates with a calculated model c-index 0.955 [14]. While some variables differ by year, such as mortality and morbidity estimates, the ACS NSQIP surgical risk calculator includes well-known variables such as age, procedure CPT, ASA class, preoperative functional status, body mass index, ascites, albumin, creatinine, and others enumerated in risk calculator and defined in ACS NSQIP Semiannual Reports. 
Table 6 Multivariable model estimating associations with discharge to a facility other than home

\begin{tabular}{lll}
\hline & OR & $95 \%$ CI \\
\hline Age & Reference & \\
$65-74$ & 2.33 & $1.90-2.86$ \\
$75-84$ & 4.75 & $3.50-6.45$ \\
85 and older & & $<0.001$ \\
Operation & Reference & $<0.001$ \\
Hepatopancreatic & 1.47 & $1.18-1.84$ \\
Colorectal & 5.46 & $4.39-6.80$ \\
ACS NSQIP-estimated probability of morbidity & 2.30 & $1.85-2.85$ \\
Mobility aid & 1.56 & $1.19-2.05$ \\
Fall history & & 0.001 \\
Consent & Reference & $<0.001$ \\
Signed by patient & 4.71 & 0.001 \\
Surrogate & & $2.78-7.97$ \\
Patient origin & Reference & \\
Lives home alone & 0.41 & $0.33-0.50$ \\
Lives with companion & 1.06 & $0.32-3.49$ \\
Lives at facility & & $<0.001$
\end{tabular}

ACS NSQIP American College of Surgeons National Surgical Quality Improvement Program

As such, these estimated probabilities already account for clinically significant and statistically appropriate patient-specific comorbid conditions in the entire ACS NSQIP patient cohort. After adjusting for these clinically relevant covariates, the four additional GSFR geriatricspecific variables were not associated with postoperative outcomes with exception of discharge destination. Importantly, patient's functional status (defined in ACS NSQIP as independent, partially dependent, and totally dependent) is already included in the ACS NSQIP-estimated probability of mortality and estimated probability of morbidity variables. At baseline condition, the estimated probabilities adjust for patients underlying functional status, arguably one of the global summary estimates for increasing age and frailty [15-17].

Discharge to a facility has important long-term implications on patient's survival and quality of life. Hospital discharge to a skilled nursing facility has been associated with poor prognosis and significantly decreased survival among patient populations who had intraabdominal operations [18], cardiovascular operations [19], or required intensive care unit admission [20]. In a study of an 89,405 patient cohort who had four types of intraabdominal operations (cholecystectomy, colectomy, hysterectomy/ oophorectomy, or prostatectomy), 1-year mortality among patients discharged to an institutional care facility was $22.2 \%$ compared to $5.9 \%$ for patients discharged home [18]. A subsequent analysis of 308,016 surgical patients from Centers for Medicare and Medicaid Services reported a $26.4 \%$ 1-year mortality among patients admitted for acute care surgical admission and discharged to a skilled nursing facility [21]. While quantitative studies examining the effect of discharge to a facility and long-term quality of life among surgical patients are lacking, the qualitative effects and patient impact have been well described [22].

Even though the ACS NSQIP-captured 30-day mortality and morbidity outcomes are not directly associated with geriatric-specific variables in this analysis, the association between every geriatric-specific variable and discharge destination is critically important. As previously highlighted long-term data demonstrate, discharge to institutional facility is associated with significantly poorer overall prognosis and survival. And while a direct link between geriatric-specific variables and 30-day morbidity and mortality is mitigated by statistical adjustment using the estimated ACS NSQIP probabilities available within the preoperative ACS NSQIP calculator, long-term effects of discharge to a facility other than home must be considered. Use of mobility aid, preoperative falls, consent signed by a surrogate, and living at home without a companion were all significantly associated with discharge to a facility.

Despite robust data collection and clinically relevant primary and secondary outcomes, this study has a number of limitations inherent to retrospective cohort database study design and analysis. Most notably, the outcome measures are limited to the 30-day ACS NSQIP follow-up 
period. Quality-of-life outcomes as well as longer-term functional improvement outcomes were not measured. In addition, individual patient's functional fitness for surgery, even among patients who are functionally independent, cannot be ascertained. A number of recent studies have described importance of fitness and potential role for prehabilitation in improvement of patient outcomes [23-25]. However, data summarized in this study demonstrate significant associations between the preoperatively defined geriatric-specific variables and postoperative discharge destination among patients selected for major abdominal hepatopancreatic or colorectal operations.

\section{Conclusions}

Patient-specific discussion of potential risks and benefits is paramount in every elective surgical setting. Numerous preoperative tests can quantify frailty, cognitive decline, and/or functional dependence. The four factors included in the ACS NSQIP GSFR geriatric-specific variables evaluated in this study are easy to quantify during any preoperative clinic visit, are significantly associated with postoperative patient discharge to a facility, and can help guide patient-surgeon decision making.

Acknowledgement American College of Surgeons National Surgical Quality Improvement Program and the hospitals participating in the ACS NSQIP are the source of the data used herein; they have not verified and are not responsible for the statistical validity of the data analysis or the conclusions derived by the authors.

Funding This study was supported in part by the L30 CA220861 Award from National Cancer Institute and Grant \#IRG 81-001-26 from the American Cancer Society to VMZ.

\section{Compliance with ethical standards}

Conflict of interest None of the authors have personal, financial, or other conflicts of interest to disclose.

\section{Appendix}

\section{ACS NSQIP Targeted Module CPT codes}

Hepatectomy: 47120, 47122, 47125, 47130.

Pancreatectomy: 48120, 48140, 48145, 48146, 48148, 48150, 48152, 48153, 48154, 48155, 48999.

Colectomy: 44140, 44141, 44143, 44144, 44145, 44146, 44147, 44150, 44151, 44160, 44204, 44205, 44206, 44207, 44208, 44210.

Proctectomy: 44155, 44156, 44157, 44158, 44211, 44212, 45110, 45111, 45112, 45113, 45114, 45116, 45119,
45120, 45121, 45123, 45126, 45130, 45135, 45160, 45395, $45397,45402,45550$.

\section{References}

1. Mohanty S, Rosenthal RA, Russell MM, Neuman MD, Ko CY, Esnaola NF (2016) Optimal perioperative management of the geriatric patient: a best practices guideline from the American College of Surgeons NSQIP and the American Geriatrics Society. J Am Coll Surg 222(5):930-947

2. Giannotti C, Sambuceti S, Signori A et al (2019) Frailty assessment in elective gastrointestinal oncogeriatric surgery: predictors of one-year mortality and functional status. J Geriatr Oncol 10(5):716-723

3. Racz J, Dubois L, Katchky A, Wall W (2012) Elective and emergency abdominal surgery in patients 90 years of age or older. Can J Surg 55(5):322-328

4. Revenig LM, Canter DJ, Taylor MD et al (2013) Too frail for surgery? Initial results of a large multidisciplinary prospective study examining preoperative variables predictive of poor surgical outcomes. J Am Coll Surg 217(4):665-670

5. Eamer G, Al-Amoodi MJH, Holroyd-Leduc J, Rolfson DB, Warkentin LM, Khadaroo RG (2018) Review of risk assessment tools to predict morbidity and mortality in elderly surgical patients. Am J Surg 216(3):585-594

6. Turrentine FE, Wang H, Simpson VB, Jones RS (2006) Surgical risk factors, morbidity, and mortality in elderly patients. J Am Coll Surg 203(6):865-877

7. Robinson TN, Wu DS, Pointer LF, Dunn CL, Moss M (2012) Preoperative cognitive dysfunction is related to adverse postoperative outcomes in the elderly. J Am Coll Surg. 215(1):12-17 (discussion 17-18)

8. Makary MA, Segev DL, Pronovost PJ et al (2010) Frailty as a predictor of surgical outcomes in older patients. J Am Coll Surg 210(6):901-908

9. Zucchelli A, Vetrano DL, Grande G et al (2019) Comparing the prognostic value of geriatric health indicators: a population-based study. BMC Med 17(1):185

10. Chow WB, Rosenthal RA, Merkow RP et al (2012) Optimal preoperative assessment of the geriatric surgical patient: a best practices guideline from the American College of Surgeons National Surgical Quality Improvement Program and the American Geriatrics Society. J Am Coll Surg 215(4):453-466

11. Berian JR, Zhou L, Hornor MA et al (2017) Optimizing surgical quality datasets to care for older adults: lessons from the American College of Surgeons NSQIP geriatric surgery pilot. J Am Coll Surg 225(6):702-712

12. American College of Surgeons National Surgical Quality Improvement P (2018) Geriatric Surgery Research File (GSRF) user guide 2014-2016. American College of Surgeons

13. Bilimoria KY, Liu Y, Paruch JL et al (2013) Development and evaluation of the universal ACS NSQIP surgical risk calculator: a decision aid and informed consent tool for patients and surgeons. J Am Coll Surg. 217(5):833-842 e831-e833

14. American College of Surgeons National Surgical Quality Improvement P (2016) ACS NSQIP ${ }^{\circledR}$ semiannual report. Chicago: American College of Surgeons; July 8, 2016

15. Simone PM, Haas AL (2013) Frailty, leisure activity and functional status in older adults: relationship with subjective well being. Clin Gerontol 36(4):275-293 
16. Ronning B, Wyller TB, Jordhoy MS et al (2014) Frailty indicators and functional status in older patients after colorectal cancer surgery. J Geriatr Oncol 5(1):26-32

17. Roppolo M, Mulasso A, Gobbens RJ, Mosso CO, Rabaglietti E (2015) A comparison between uni- and multidimensional frailty measures: prevalence, functional status, and relationships with disability. Clin Interv Aging 10:1669-1678

18. Legner VJ, Massarweh NN, Symons RG, McCormick WC, Flum DR (2009) The significance of discharge to skilled care after abdominopelvic surgery in older adults. Ann Surg 249(2):250-255

19. Edgerton JR, Herbert MA, Mahoney C et al (2013) Long-term fate of patients discharged to extended care facilities after cardiovascular surgery. Ann Thorac Surg 96(3):871-877

20. Rady MY, Johnson DJ (2004) Hospital discharge to care facility: a patient-centered outcome for the evaluation of intensive care for octogenarians. Chest 126(5):1583-1591

21. Hakkarainen TW, Arbabi S, Willis MM, Davidson GH, Flum DR (2016) Outcomes of patients discharged to skilled nursing facilities after acute care hospitalizations. Ann Surg 263(2):280-285
22. Mor V, Intrator O, Feng Z, Grabowski DC (2010) The revolving door of rehospitalization from skilled nursing facilities. Health Aff (Millwood) 29(1):57-64

23. Dunne DF, Jack S, Jones RP et al (2016) Randomized clinical trial of prehabilitation before planned liver resection. Br J Surg 103(5):504-512

24. Moran J, Guinan E, McCormick P et al (2016) The ability of prehabilitation to influence postoperative outcome after intraabdominal operation: a systematic review and meta-analysis. Surgery 160(5):1189-1201

25. Myers JN, Fonda H (2016) The impact of fitness on surgical outcomes: the case for prehabilitation. Curr Sports Med Rep 15(4):282-289

Publisher's Note Springer Nature remains neutral with regard to jurisdictional claims in published maps and institutional affiliations. 